\title{
Three-dimensional bioprinting and tissue fabrication: prospects for drug discovery and regenerative medicine
}

This article was published in the following Dove Press journal:

Advanced Health Care Technologies

19 August 2015

Number of times this article has been viewed

\author{
Vivian K Lee ${ }^{1,2}$ \\ Guohao Dai ${ }^{1,2}$ \\ 'Department of Biomedical \\ Engineering, ${ }^{2}$ Center for \\ Biotechnology and Interdisciplinary \\ Studies, Rensselaer Polytechnic \\ Institute, Troy, NY, USA
}

\begin{abstract}
Bioprinting technology has emerged as a powerful tool for building tissue and organ structures for drug discovery and regenerative medicine applications. In general, bioprinting uses a computer-controlled three-dimensional (3D) printing device to accurately deposit cells and biomaterials into precise geometries with the goal of creating anatomically correct biological structures. While traditional 3D printing uses metals, plastics, and polymers as printing materials or "ink", bioprinting deals with living cells and biological matrix. Hence, there are significant challenges to make a transition from traditional 3D printing to bioprinting, and ultimately achieve functional outcomes in bioprinted tissues. Therefore, it is critical that there is new technology development and in-depth basic research in bioprinted tissues, such as developing novel biomaterials specifically for use in bioprinting and biofabrication techniques, understanding the cell-matrix remodeling for the desired mechanical properties, and functional outcomes, establishing proper vascular perfusion, etc. Currently, there is active research going on bioprinting technology and its potential as a future source for tissue implants. This review paper overviews the current state of the art in bioprinting technology and focuses on the outcomes of the bioprinted tissues and their potential applications in drug discovery and regenerative medicine. Current challenges and limitations are highlighted, and future directions for next-generation bioprinting technology are also presented.
\end{abstract}

Keywords: 3D bioprinting, vascularization, tissue regeneration, 3D tissue model, biofabrication, regenerative medicine

\section{Introduction}

Three-dimensional (3D) printing, also called additive manufacturing or solid freeform fabrication, is driving major innovations in many areas, such as engineering, manufacturing, art, design, and education. 3D printing systems build 3D structures by layering materials onto a moving platform. Commercially available systems utilize one of following processes: 1) photopolymerization of liquid monomer, 2) sintering powdered materials, 3 ) thermal or chemical process as material passes through a nozzle, or 4) printing materials such as chemical binder onto powder. 3D printing techniques can be easily automated and integrated with imaging techniques to produce scaffolds that are customized in size and shape for specific applications. 3D printing has been very successful in making biomaterial scaffolds, and is becoming an important enabling technology for tissue engineering. ${ }^{1-8}$ However, the translation of $3 \mathrm{D}$ printing into building living tissue still faces significant challenges. Typically, toxic solvents, high temperatures, or strong ultraviolet (UV) light are still widely used in most 3D printing techniques, making them not suitable to build live tissues and limiting their further applications in tissue engineering.
Correspondence: Guohao Dai Department of Biomedical Engineering, Center for Biotechnology and Interdisciplinary Studies, Rensselaer Polytechnic Institute, Room 3123, 110 8th Street, Troy, NY 12180 , USA

Tel +I 5 I8 2764476

Fax +I 5182763035

Email daig@rpi.edu 
To engineer 3D live tissues, many groups around the world have begun to develop technology to simultaneously deposit hydrogels with live cells to form 3D tissue structures. ${ }^{9-16}$ This new concept, also called organ printing or bioprinting, is an advanced form of $3 \mathrm{D}$ printing. The main features of this technology include the use of phasechanging hydrogels without harsh chemicals and a dispensing technology that is gentle to the cells. Current strategies for inducing phase change (from liquid to solid form after printing) include UV light, temperature, $\mathrm{pH}$, and ion concentrations, which can be used on a variety of natural and synthetic hydrogels. An important advantage of this process is the ability to simultaneously deposit live cells and growth factors along with biomaterial scaffolds at the accurate location to mimic the native tissue architecture and formation process. $3 \mathrm{D}$ bioprinting has great potentials to 1) create fully functional replacements for damaged tissues in patients and 2) rapidly fabricate small-sized human-based tissue models or organoids, for applications in diagnostics, disease modeling, and drug development. Despite all the promises, the usage of this technology in tissue engineering is still at its infancy. There are significant challenges that must be solved before it can generate a major impact to the field. Compared with nonbiological printing, 3D bioprinting involves additional complexities, such as the choice of materials, cell types, growth and differentiation factors, and technical challenges related to the sensitivities of living cells and the construction of tissues. Addressing these complexities requires the integration of technologies from the fields of engineering, biomaterials science, cell biology, physics, and medicine. This review summarizes the most recent development of $3 \mathrm{D}$ bioprinting of live tissues, with emphasis on the functional consequences and/or in vivo applications of the bioprinted tissues.

\section{Bioprinting techniques}

Currently, there are three major types of 3D bioprinting techniques: inkjet bioprinting, microextrusion bioprinting, and laser-assisted bioprinting (LAB; Figure 1). ${ }^{17}$ Each of these approaches has been used widely and has many advantages and drawbacks (Table 1).

\section{Inkjet 3D bioprinting}

Inkjet-based bioprinting is a noncontact technique in which droplets of cells or biomaterials are dispensed, driven either by thermal bubble, piezoelectric actuator, or electromechanical valve-controlled pressure pulse. The thermal printers cause electric heating of the print head to produce pressure pulses that force droplets out of the nozzle. The local heating generates a bubble in the bioink chamber and ejects a small droplet. The localized heating in thermal printers can be very high, ranging from $200^{\circ} \mathrm{C}$ to $300^{\circ} \mathrm{C}$, but it lasts for a very short duration of $2 \mu \mathrm{s}$; thus, the overall temperature increases only by a maximum of $4^{\circ} \mathrm{C}-10^{\circ} \mathrm{C}$ and usually does not exert any significant impact on the viability of cells. ${ }^{18,19}$ While thermal inkjet printers are of low cost, readily available, and have high print speeds, they suffer from the disadvantages such as the lack of precise directionality and size control of droplet, thermal and mechanical stress applied on cells and biomaterials, frequent nozzle clogging, and unreliable cell encapsulation. The piezoelectric 3D bioprinters contain a piezoelectric crystal, which is in response to applied voltage,

\section{Inkjet printing}

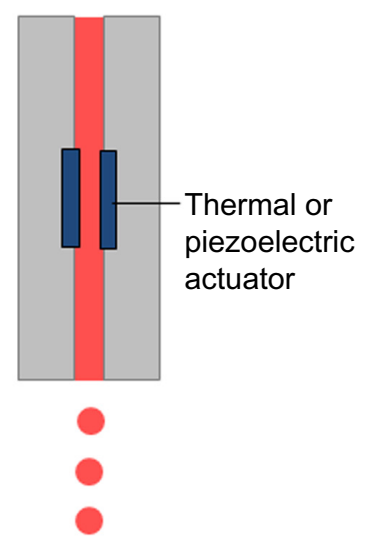

\section{Micro-extrusion}

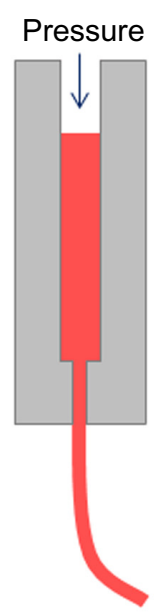

\section{Laser printing}

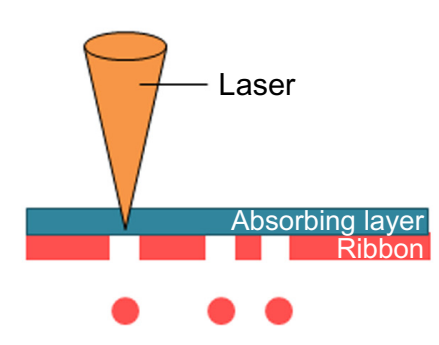

Figure I Schematics of the major bioprinting mechanisms. 
Table I Comparison of major bioprinting techniques

\begin{tabular}{|c|c|c|c|}
\hline Category & Inkjet & Microextrusion & Laser printing \\
\hline Material viscosity $(\mathrm{mPa} / \mathrm{s})$ & Low (3-12) & High $\left(30-6 \times 10^{7}\right)$ & $\mathrm{I}-300$ \\
\hline Gelation/cross-linking methods & $\begin{array}{l}\text { Chemical or photo-cross-linking, } \\
\text { temperature }\end{array}$ & $\begin{array}{l}\text { Chemical or photo-cross-linking, } \\
\text { temperature }\end{array}$ & Chemical or photo-cross-linking \\
\hline Print speed & I-I0,000 droplets/s & $10-50 \mu \mathrm{m} / \mathrm{s}$ & $200-1600 \mathrm{~mm} / \mathrm{s}$ \\
\hline Resolution & 50-300 $\mu \mathrm{m}$ wide droplets & $\mathrm{I} 00 \mu \mathrm{m}$ to I $\mathrm{mm}$ wide & $50 \mu \mathrm{m}$ \\
\hline Accuracy & Medium & Medium-low & High \\
\hline Cell viability & $>85 \%$ & $40 \%-80 \%$ & $>85 \%$ \\
\hline Cell density & $10^{6}-10^{7}$ cells $/ \mathrm{mL}$ & High: cell spheroids & $10^{6}-10^{7}$ cells $/ \mathrm{mL}$ \\
\hline Biomaterials used & $\begin{array}{l}\text { Hydrogels, fibrin, agar, collagen, } \\
\text { alginate }\end{array}$ & $\begin{array}{l}\text { Hyaluronic acid, gelatin, alginate, } \\
\text { collagen, fibrin }\end{array}$ & Hydrogels, nano-hydroxyapatite \\
\hline Mechanical/structural integrity & Low & High & Low \\
\hline Fabrication time & Medium & Short & Long \\
\hline Scalability & Yes & Yes & Limited \\
\hline Cost & Low & Medium & High \\
\hline Example applications & Skin, ${ }^{22,53}$ vascular, ${ }^{65,66,69}$ cartilage ${ }^{46,78,79}$ & Trachea, ${ }^{38,71}$ cardiac valve ${ }^{75-77}$ & Skin $^{54,55}$ \\
\hline
\end{tabular}

induces a rapid change in shape and creates an acoustic wave inside the print head. This acoustic wave helps breaking the liquid present inside the print head into many droplets and the ejection of droplets at regular intervals. ${ }^{20}$ Recently, a newer technology was developed to generate droplets using gentle acoustic field. A variety of cells can be encapsulated in acoustic picoliter droplets of $\sim 30 \mu \mathrm{m}$ in diameter at rates from 1 to 10,000 droplets per second. The benefits of this technology include high precision, high viability, and controlled directionality. ${ }^{21}$ Another method of inkjet bioprinting is by electromechanical valve-controlled pressure pulse. In this method, a constant pressure is applied to the liquid contained in the nozzle, and the opening and closing of the electromechanical valve under the control of pulsed voltage $(\sim 100-500 \mu \mathrm{s})$ will lead to droplet formation. Compared to thermal inkjet printers, this method does not lead to the heating of the cells, thus allowing very gentle deposition of the cells and high post-printing viability. ${ }^{22}$

Advantages of an inkjet printer include low cost and versatility. Inkjet-based 3D bioprinting methods can generate relatively high-resolution structures $(20-100 \mu \mathrm{m})$. The drop size (from 1 to $300 \mathrm{pL}$ ) and drop deposition rate (1-10,000 droplets/s) can be controlled electronically. ${ }^{23}$ Owing to the dispensing mechanisms and noncontact nature of the inkjet printer, low-viscous materials (viscosity $<10 \mathrm{cP}$ ) are preferably used, whereas high-viscosity materials cannot be effectively dispensed by inkjet printer. As a result, the printed structure often has weak mechanical properties. ${ }^{22,24}$ The inkjet bioprinting not only facilitates the deposition of primary cells and/or stem cells with required density, but also maintains high cell viability and function after printing. While these features demonstrate the potential of inkjet-based
3D bioprinting for the production of functional structures, addressing the abovementioned drawbacks can certainly make this technique an invaluable tissue engineering tool.

\section{Microextrusion bioprinting}

Microextrusion 3D bioprinters produce continuous beads of material that are deposited in two dimensions via a syringe nozzle in which the viscous materials are squeezed out under constant pressure. The deposited layer serves as a foundation for the subsequent layer, while the stage or microextrusion head is moved along the z-axis, finally resulting in the formation of a 3D structure. The amount of dispensed cellladen hydrogel can be adjusted by 1) controlling the level of pneumatic pressure or mechanical pressure created by the displacement of the piston of the syringe pump, 2) the nozzle size, and 3 ) the nozzle moving speed. ${ }^{25}$ Following $2 \mathrm{D}$ pattern printing of the hydrogels, these are solidified and stacked layer by layer to form 3D structures.

Microextrusion bioprinting is relatively simple to construct and is affordable. High-viscosity biomaterials such as hydrogels, biocompatible copolymers, and cell spheroids can be printed through the micro-nozzle, and microextrusion bioprinter can easily create large constructs by using larger extruders. In addition, the capability to deposit very high cell densities is an advantage of microextrusion 3D bioprinting. Multicellular cell spheroids, which possess the mechanical and functional properties of the tissue extracellular matrix (ECM), can be deposited to self-assemble into 3D structure by the microextrusion printers. ${ }^{26}$ This strategy of the self-assembling spheroids can potentially accelerate tissue organization and the formation of complex structures without the need for additional scaffolds. The disadvantage 
of microextrusion bioprinting is that only materials with high viscosity can be extruded. This results in high shear stress, which tends to kill the cells during the printing process. Most reported studies showed that cell survival rates are generally lower than those seen with the inkjet printers, in the range of $40 \%-86 \%$, with the survival rate decreasing with increasing extrusion pressure. ${ }^{27}$

\section{Laser-assisted bioprinting}

LAB is also called laser-assisted direct writing, which uses the energy of pulsed laser to induce the transfer of materials. ${ }^{28}$ A laser-assisted 3D bioprinter consists of 1) a pulsed laser beam with a focusing system, 2) a "ribbon" that has a donor transport support, typically made from glass covered with a laser-energy-absorbing layer (eg, gold or titanium) and a layer of biological material containing cells and/or hydrogel, and 3 ) a receiving substrate facing the ribbon. Laser-assisted $3 \mathrm{D}$ bioprinter focuses laser pulses on the absorbing gold layer of the ribbon and this generates a high-pressure bubble, which in turn propels cell-containing materials toward the collector substrate. LAB can deposit cells at a density of up to $10^{8}$ cells $/ \mathrm{mL}$ with the resolution of a single cell per drop using a laser pulse at high speed..$^{29,30}$ These features allow LAB to create high-throughput laser patterning of cells and biomaterials. Although this technique is able to produce relatively higher resolution patterns, it results in lower cell viability in the printed hydrogel in comparison to other inkjet mechanisms. LAB is a nozzle-free technique, and therefore does not have the problems of nozzle clogging with cells or materials, which are major drawbacks of other bioprinting technologies. Another advantage of LAB is its compatibility with a wide range of biomaterial viscosities $(1-300 \mathrm{mPa} / \mathrm{s})$.

\section{Biomaterials for bioprinting}

Biomaterials are responsible for supporting the cellular components during and after bioprinting procedures, and thus play a significant role in successful implementation of bioprinting. Biomaterials for bioprinting have to meet several criteria including cytocompatibility, printability, ease of phase transition, and compatibility of bioreactors. Currently, most bioprinting applications use or modify the same biomaterials used in traditional tissue engineering, and there is no ideal material specially designed for bioprinting purpose. ${ }^{31}$

The choice of printable biomaterials is mostly determined by the printability, rheological properties, and gelation/cross-link mechanisms. Because of this reason, the availability of biomaterials that are suitable for bioprinting is very limited. In the context of bioprinting, biomaterials generally fall into two primary categories. The first category is curable polymers that form mechanically robust and durable materials after solidification, providing structure and scaffolding to printed constructs. Many such materials typically require high temperatures or toxic solvents to facilitate printing and therefore are not appropriate for printing together with cells. For this reason, cells are usually seeded onto the scaffolds created from these materials after fabrication, thus avoiding conditions harmful to the cells. The second category of biomaterials is soft materials, generally with high water content, inside of which cells are capable of residing. Soft materials can comprise synthetic or natural polymers, but do not possess the same levels of mechanical properties as curable support polymers. The inherent characteristics of these different printing materials, including mechanical properties, melting points, and available chemistries for cross-linking and functionalization, make up the factors responsible for successful bioprinting.

For live cells, the primary biomaterial used for printing is hydrogel. ${ }^{32,33}$ Hydrogels are composed of polymer or peptide chains, and are cross-linked to form a macromolecular network after printing as a liquid precursor. Hydrogel biomaterials fall into one of two major categories: synthetic hydrogels, which employ polymers that are synthesized in the laboratory, or naturally derived hydrogels, which are purified from natural sources and are often further manipulated in the laboratory. To be considered cell-supportive, these hydrogels must not induce toxicity in cells and should provide cellbinding motifs to allow cell adherence. With the exception of the stiffest tissue types such as bone and teeth, hydrogels can recapitulate a range of elastic modulus values through manipulation of chemistry, cross-linking density, and polymer concentration, thus mimicking the elastic moduli of most soft tissues in the body. Processing techniques to generate cross-linking reactions can be designed to be noncytotoxic, allowing 3D encapsulation of cells within the hydrogel polymer networks at the time of gelation.

Common examples of synthetic hydrogels include polyethylene glycol (PEG)-based materials, such as PEG diacrylate (PEGDA), and polyacrylamide (PAAm)-based gels. Examples of naturally derived materials that are commonly used in the laboratory include collagen, hyaluronic acid, alginate, and fibrin. ${ }^{34-36}$ An overview of some of the traditional materials and variations of these materials used in bioprinting is summarized in Table 2. Typically, with synthetic 
materials, one can have more control over molecular weights and their distributions, as well as cross-linking densities, allowing for precise control of specific mechanical properties such as elastic modulus. On the other hand, naturally derived hydrogels may be more difficult to manipulate into specific ranges of physical properties, but often have an innate bioactivity through naturally occurring peptide sequences or conformational motifs that cells can interact with, supporting cell and tissue integration and biocompatibility. ${ }^{37}$

Regardless of these advantages and disadvantages, neither the synthetic nor the naturally derived hydrogels can replicate the complex composition and architectures of native ECM, and thus are unable to reconstitute the intrinsic cellular morphologies and functions. To overcome this problem, decellularized matrix components have been recently considered as a new bioink type. Park et al developed a method for bioprinting of cell-laden constructs with novel decellularized ECM (dECM) bioink. ${ }^{38}$ The tissue-specific dECM bioinks demonstrate the versatility and flexibility in creating tissuespecific bioprinted structures such as adipose, cartilage, and heart tissues. The dECM bioink is able to provide crucial cues for cell engraftment, survival, and long-term function. Using this dECM bioink in the bioprinting method, they achieved high cell viability and functionality of the printed tissue. ${ }^{39}$
Besides these synthetic and natural-derived bioinks, another category of materials that can be printed is cellaggregate-based bioink, which can be tissue spheroids, cell pellets, or tissue strands. These aggregated bioinks can be considered as "living materials" with certain measurable, transforming, and potentially controllable material properties. $3 \mathrm{D}$ functional living macrotissues and organ constructs can be constructed using tissue spheroids as building blocks. Tissue spheroids placed in close proximity undergo tissue fusion or tissue self-assembly process to form tissues. ${ }^{10}$

\section{Bioprinted tissues}

To date, a complete fully functional human-sized organ has not been printed. This remains the primary long-term goal of bioprinting research and development. However, small-scale simplified tissues are currently being created to mimic native tissues such as bone, cartilage, skin, nerve, and complex organs such as teeth, nose, ears, heart, and liver. These bioprinted tissues can be used in a variety of applications, including tissue regeneration, pathology modeling, drug development, and toxicology screening. In this section, we review some examples of the bioprinted tissues and focus on the method and their biological outcomes.

Table 2 List of commonly used biomaterials for bioprinting

\begin{tabular}{|c|c|c|c|c|c|}
\hline Materials & $\begin{array}{l}\text { Synthetic } \\
\text { or natural }\end{array}$ & Gelation mechanisms & Gelation speed & Advantages & Disadvantages \\
\hline $\begin{array}{l}\text { Acrylated PEG or } \\
\text { multi-arm PEG }\end{array}$ & Synthetic & Photopolymerization & Seconds-minutes & $\begin{array}{l}\text { Easy to control mechanical } \\
\text { properties }\end{array}$ & $\begin{array}{l}\text { No biological activities, } \\
\text { needs modification }\end{array}$ \\
\hline Collagen & Natural & $\mathrm{pH}$ and temperature & Hours & $\begin{array}{l}\text { Natural bioactivity, major } \\
\text { component of native ECM }\end{array}$ & $\begin{array}{l}\text { Slow gelation, lacks } \\
\text { mechanical properties }\end{array}$ \\
\hline $\begin{array}{l}\text { Thiolated } \\
\text { hyaluronic acid }\end{array}$ & Natural & $\mathrm{pH}$-mediated Michael addition & Minutes-hours & $\begin{array}{l}\text { Commercially available in } \\
\text { kit with gelation for cell } \\
\text { adherence }\end{array}$ & Weak mechanical properties \\
\hline $\begin{array}{l}\text { Thiolated } \\
\text { hyaluronic acid }\end{array}$ & Natural & Photopolymerization thiol-ene & Seconds & $\begin{array}{l}\text { Easily controllable, fast } \\
\text { gelation }\end{array}$ & Weak mechanical properties \\
\hline $\begin{array}{l}\text { Methacrylated } \\
\text { hyaluronic acid }\end{array}$ & Natural & Photopolymerization & Minutes & $\begin{array}{l}\text { Gelation speed modulated } \\
\text { by UV intensity }\end{array}$ & Weak mechanical properties \\
\hline $\begin{array}{l}\text { Tyramine } \\
\text { hyaluronic acid }\end{array}$ & Natural & $\begin{array}{l}\text { Chemical cross-linking (oxidative } \\
\text { coupling, tyramine- }-\mathrm{H}_{2} \mathrm{O}_{2} \text { ) }\end{array}$ & Seconds & Fast gelling & $\begin{array}{l}\text { Difficult to control geometry } \\
\text { due to fast gelation }\end{array}$ \\
\hline Gelatin & Natural & Temperature & Minutes-hours & Natural cell adherent & Unstable \\
\hline $\begin{array}{l}\text { Methacrylated } \\
\text { gelatin }\end{array}$ & Natural & Photopolymerization & Minutes & Natural cell adherent & Weak mechanical properties \\
\hline Alginate & Natural & Chemical cross-linking $\left(\mathrm{Ca}^{2+}\right)$ & Seconds & $\begin{array}{l}\text { Easy to create gel } \\
\text { microspheres }\end{array}$ & $\begin{array}{l}\text { Difficult to control } \\
\text { geometry, no cell adherent } \\
\text { without modification }\end{array}$ \\
\hline Fibrin & Natural & $\begin{array}{l}\text { Enzymatic reaction (thrombin- } \\
\text { fibrinogen) }\end{array}$ & Seconds & Cell adherent & Difficult to control geometry \\
\hline PCL & Synthetic & Melt by high temperature & Seconds & $\begin{array}{l}\text { Robust mechanical } \\
\text { properties }\end{array}$ & $\begin{array}{l}\text { High temperature, } \\
\text { incompatible with cells }\end{array}$ \\
\hline
\end{tabular}

Abbreviations: ECM, extracellular matrix; PCL, polycaprolactone; PEG, polyethylene glycol; UV, ultraviolet. 


\section{Bone}

Bone has relatively good native regeneration and healing capacity if the defect is small. Therefore, providing a porous scaffold material plus growth factors without cells is sufficient to induce osteoinductive activities when implanted in vivo in this case. There are extensive studies on the use of 3D-bioprinted bone scaffolds, such as incorporating osteogenic factors (such as bone morphogenetic proteins) in 3D-printed scaffolds, and the development of osteoinductive 3D scaffolds. ${ }^{40}$ However, large-sized bone defects hardly heal without cell delivery, hence introducing cells within the scaffolds is necessary. When 3D-printed highly porous biphasic calcium phosphate bone implants were ectopically implanted in the back of rats, osteogenic cells tended to induce bone formation mainly in the central region of the scaffolds. A combination of osteoblasts seeding and bone morphogenetic protein-2 (BMP-2) encapsulation synergistically enhanced bone formation. ${ }^{41}$ Bone marrow-derived mesenchymal stem cells (MSCs) are often used to enhance the bone formation in 3D-printed scaffolds. In another study, CD117+ (stem cell growth factor receptor) human bone marrow MSCs were dispersed in different hydrogel mixtures and applied in a 3D-printed $\beta$-TCP/PLGA (beta-tricalcium phosphate/ poly(lactic-co-glycolic acid)) scaffold. Constructs were implanted subcutaneously into nude mice for 6 weeks. It was demonstrated that biomechanical stiffness, radiological densities, and bone ECM accumulation were significantly enhanced after 6 weeks. ${ }^{42}$

Using 3D printing, an anatomically shaped scaffold can be created to match the actual defect of patients based on the medical imaging data. In one study, anatomically shaped polycaprolactone (PCL) scaffolds with varying porosities were used to support the induction of human adipose-derived stem cells to form vascularized bone to repair mandibular and maxillary bone defects (Figure 2A). ${ }^{43}$ The findings demonstrated the capabilities and potential of 3D-bioprinted scaffolds to create anatomically shaped, vascularized bone grafts.

To improve the functionality of the bioprinted structures, multicellular printed constructs were created and demonstrated the heterogeneity similar to that of the native tissues. A porous construct containing spatially organized osteogenic and endothelial progenitor cells was printed and subcutaneously implanted in immune-deficient mice. ${ }^{44}$ It was observed that perfused blood vessels formed in the endothelial progenitor cell-laden compartment and new bone formation was taking place in the multipotent stromal cell-laden part of the printed constructs. This study illustrated that bioprinting can be used to create different patterns of cell differentiation according to the deposited progenitor cell type, which is valuable to achieve the heterogeneity of the desired tissue structures.
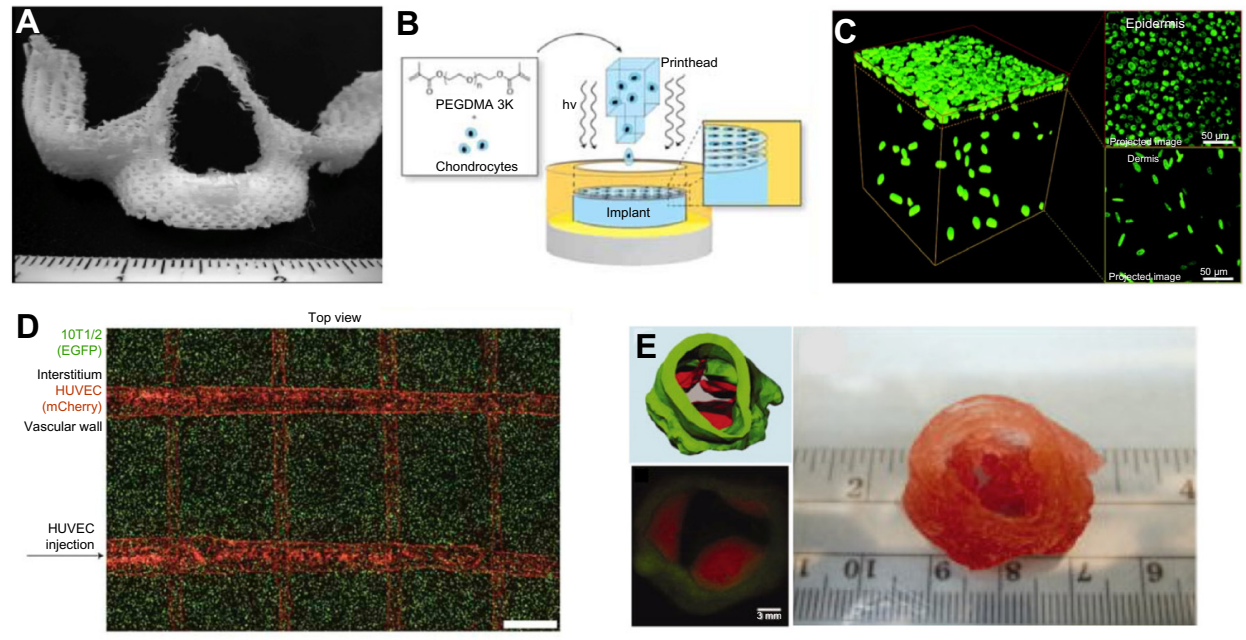

Figure 2 Examples of the bioprinted tissues.

Notes: (A) 3D-printed, porous PCL scaffolds of maxilla at 40\% infill density. Copyright @ 20I4. John Wiley and Sons. Reproduced from Temple JP, Hutton DL, Hung BP, et al. Engineering anatomically shaped vascularized bone grafts with hASCs and 3D-printed PCL scaffolds. J Biomed Mater Res A. 20I4; I02(I2):43 I7-4325 ${ }^{43}$ (B) Schematic of bioprinting cell-laden constructs for cartilage tissue engineering with simultaneous photopolymerization process. Copyright (C) 20I2. John Wiley and Sons. Reproduced from Cui X, Breitenkamp K, Lotz M, D'Lima D. Synergistic action of fibroblast growth factor-2 and transforming growth factor-betal enhances bioprinted human neocartilage formation. Biotechnol Bioeng. 2012; 109(9):2357-2368. ${ }^{47}$ (C) 3D-printed skin tissue with keratinocyte-populated epidermal layer and fibroblast-populated dermal layer. Scale bar, $500 \mu \mathrm{m}$. Copyright (C) 20I4, Mary Ann Liebert, Inc. Reproduced from Lee V, Singh G, Trasatti JP, et al. Design and fabrication of human skin by three-dimensional bioprinting. Tissue Eng Part C Methods. 20I4;20(6):473-484..$^{53}$ (D) 3D vascular network (red) with I0T I/2 cells (green) in the interstitial space. Scale bar, I mm. Reprinted by permission from Macmillan Publishers Ltd: Nature Materials. Miller JS, Stevens KR, Yang MT, et al. Rapid casting of patterned vascular networks for perfusable engineered three-dimensional tissues. Nat Mater. 20I2; I I(9):768-774, copyright @ 2012.61 (E) Bioprinting of aortic valve conduit with dual cell types for valve root (green, smooth muscle cells) and valve leaflets (red, valve leaflet interstitial cells). Copyright @ 2013 . John Wiley and Sons. Reproduced from Duan B, Hockaday LA, Kang KH, Butcher JT. 3D bioprinting of heterogeneous aortic valve conduits with alginate/gelatin hydrogels. J Biomed Mater Res A. 20 I 3; I0 I (5): I 255-1264. ${ }^{75}$

Abbreviations: HUVEC, human umbilical vein endothelial cell; PCL, polycaprolactone; PEGDMA, polyethylene glycol dimethacrylate; 3D, three-dimensional; EGFP, enhanced green fluorescent protein. 


\section{Cartilage}

Cartilage is an aneural, avascular tissue that contains few cells. Articular cartilage is a heterogeneous tissue with a composition that varies in different regions. Each zone differs in cell density, glycosaminoglycan contents, biosynthetic activities, and mechanical properties. Cells in different zones also differ in size and morphology. Because of these unique properties, 3D bioprinting technology is particularly advantageous in creating the complex $3 \mathrm{D}$ zonal ECM structure. It can be achieved by controlling each printing layer with varying biomaterial properties and cell types.

Recently, a hybrid inkjet printing system using off-theshelf components was reported to produce cartilage constructs with improved biological and mechanical properties. Electrospun fibers were alternated with inkjet-printed rabbit elastic chondrocytes suspended in a fibrin-collagen hydrogel. The fabricated constructs formed ectopic cartilage-like tissue after subcutaneous implantation in mice. The depositions of type II collagen, which is specific to cartilage, and glycosaminoglycans were observed, and the construct demonstrated improvement of mechanical properties over alginate or fibrin-collagen gels alone ${ }^{45}$ The feasibility of fabricating anatomic cartilage structures was shown by delivering chondrocytes and PEG dimethacrylate to precise target locations in 3D for mimicking different zones of articular cartilage (Figure 2B). ${ }^{46,47}$ In another study, a 3D fiber deposition technique was used to fabricate cell-laden, heterogeneous hydrogel constructs for potential use as osteochondral grafts. The porosity and elastic modulus of the scaffold were varied by changing fiber deposition spacing or angle. Human chondrocytes and osteogenic progenitors were incorporated within different regions to mimic the zonal structure of osteochondral tissue. ${ }^{48}$ Distinctive tissue formation was observed, both in vitro after 3 weeks and in vivo at different locations within one construct. However, the integration of cartilage grafts with the surrounding native articular cartilage in long-term preclinical or clinical studies remains a challenge, regardless of the use of 3D printing for scaffold fabrication.

MSCs have also been introduced in the 3D-bioprinted construct for cartilage tissue engineering. A composite of

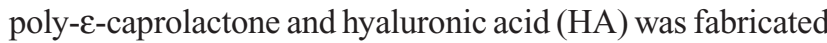
and seeded with human bone marrow stem cells (hBMSCs). An overlaying layer of PEG-based hydrogel encapsulating hBMSCs or hBMSC-derived chondrocytes was constructed in anatomic shape, and subsequently implanted subcutaneously in athymic rats. After 6 weeks, hBMSCs presented significantly more angiogenesis, whereas hBMSC-derived chondrocytes produced more mineralized tissue in microchannels and glycosaminoglycan matrix in the cartilage-like layer. ${ }^{49}$
The ability to generate precise spatial patterns is one of the advantages of 3D bioprinting, and can be applied to create zonal gradient of the cartilage. It is demonstrated that engineering anisotropic biomimetic fibrocartilage microenvironment is feasible using 3D bioprinting. For example, the biochemical gradient is created by bioprinting nanoliter droplets encapsulating human MSCs, bone morphogenetic protein-2, and transforming growth factor beta 1 (TGF $\beta 1$ ) to mimic an anisotropic biomimetic fibrocartilage microenvironment. The incorporated biochemical factors presented multiphasic anisotropic structure after patterning. This construct displayed simultaneous differentiation of MSC populations into osteogenic and chondrogenic phenotype within a single construct. The differentiation results were confirmed by the upregulation of osteogenesis- and chondrogenesis-related genes. ${ }^{50}$

3D-bioprinted constructs have also demonstrated some in vivo success in repairing cartilage defects. ${ }^{51,52}$ In this study, they fabricated an anatomically similar bioscaffold via layerby-layer bioprinting to replace the humeral head of the rabbit forelimb joint, an implant with spatial delivery of TGF 33 . After 4 months of implementation, the articular surface of TGF 33 -infused scaffolds was fully covered with hyaline cartilage and integrated with regenerated subchondral bone that contained well-developed blood vessels. The scaffold also presented mechanical properties similar to that of native articular cartilage. Subsequent study showed that TGF $\beta 3$ and stromal-derived factor-1 (SDF-1) not only chemotactically recruited adipose stem/progenitor cells, bone marrow MSCs, and synovial stem cells, but also induced chondrogenesis of the recruited cells, suggesting that homing of multiple stem/progenitor cell populations may potentially serve as an alternative or adjunctive approach to cell transplantation for cartilage in clinical applications. ${ }^{51,52}$

\section{Skin}

In skin injuries, autograft, allograft, wound dressing, and tissue-engineered substitutes are the current treatment choices. Unlike traditional tissue engineering strategies, 3D bioprinting of skin takes into account subtle cell-cell interactions as well as cell-matrix interactions and precise cell layer positioning. In addition to live cells, soluble molecules and phase-changing hydrogels can also be dispended in 3D-printed skin grafts. Lee et al developed a skin biofabrication method using an inkjet-based bioprinter (Figure 2C). Collagen precursor, keratinocytes, and fibroblasts were alternatively printed in a layer-by-layer manner, so that it was possible to construct biomimetic skin with high viability. After air-liquid interface culture, the 3D-bioprinted skin showed 
similar morphology and histology to native skin layers prior to in vivo implantation. ${ }^{22,53}$

Koch et al fabricated 3D skin grafts through deposition of fibroblasts and keratinocytes embedded in collagen, using a LAB technique, ${ }^{54}$ which resulted in the formation of the basement membrane and intercellular junctions. These skin constructs were subsequently tested in vivo, employing the dorsal skin chamber in nude mice. The constructs were placed into full-thickness skin wounds, which became fully integrated with the surrounding tissue after 11 days of implementation. The printed keratinocytes formed a multilayer-stratified epidermis with stratum corneum. E-cadherin as an indicator for adherens junctions and tissue formation could be found in the epidermis in vivo as well as in vitro. In mice, some level of angiogenesis could be found in the direction toward the printed grafts from the wound bed and the wound edges, suggesting the integration of the bioprinted skin with the host tissue. ${ }^{55}$

Bioprinting can also be used directly onto the wounded area in situ. In one study, bioprinting technology was employed to treat full-thickness skin wounds in mice. Amniotic fluid-derived stem (AFS) cells and bone marrowderived MSCs were suspended in fibrin-collagen gel and "printed" over the wound site. At days 0, 7, and 14, AFS celland MSC-driven constructs presented significantly higher level of wound closure and re-epithelialization compared to those treated with only fibrin-collagen gel. Histology showed that microvessel density and capillary diameters were greater in the AFS cell-treated wounds than the MSC-treated wounds, whereas the skin treated only with gel showed the lowest amount of microvessels. These results indicate that bioprinting AFS cells could be an effective treatment for large-scale wounds and burns. ${ }^{56}$

\section{Vascular}

The integration of vascular structure in tissue-engineered constructs remains a significant challenge. Without proper vascular supplies, it is difficult to achieve tissue constructs beyond several hundred micrometer thickness in the bioprinted tissues. Several methods are currently being investigated utilizing bioprinting techniques to form vascular channels. These methods include sacrificial channels ${ }^{57}$ that can later be seeded with endothelial cells, lines of endothelial cells ${ }^{23}$ that can later be remodeled into endothelial tubes, and free-standing tubular structures ${ }^{9}$ formed using several bioprinting techniques. Norotte et al have used bioprinting to print cell aggregates in a scaffold-free substrate to form branched vessels and have demonstrated that the cells will remodel and form a construct similar to a blood vessel. ${ }^{9}$ Another study used 3D bioprinting to create vessel-like constructs using hyaluronan hydrogels cross-linked with tetrahedral PEG tetracrylates. ${ }^{58}$

Bioprinting is combined with other microtechnologies and materials to form vascular channels. To fabricate embedded vasculature during bioprinting, Kolesky et al developed an aqueous fugitive ink composed of Pluronic F127 that can be easily printed and removed under mild conditions. Pluronic F127 is biologically inert to multiple cell types over the short time periods of the fabrication process. This material undergoes thermally reversible gelation above a critical temperature, which can be integrated into the printing process to construct vascular network and subsequently melted. They synthesized gelatin methacrylate (GelMA) for use as a bulk matrix and cell carrier. GelMA is a denatured collagen that is modified with photopolymerizable methacrylate groups, allowing the matrix to be covalently cross-linked by UV light after printing. ${ }^{59}$ They included cells in GelMA and printed the structure under the control of UV light, together with the Pluronic F127-generated vascular channels. Thus, a vascularized, heterogeneous cell-laden tissue construct can be created using 3D bioprinting. In another study, 3D micro-molding technique was used to print agarose template fibers to fabricate microchannel networks with various architectural features within photo-cross-linkable GelMA hydrogel constructs. Using this approach, functional and perfusable microchannels inside GelMA hydrogels were created. Functionality of the fabricated vascular networks was demonstrated to improve mass transport, cellular viability, and differentiation within the cell-laden tissue constructs. ${ }^{60}$

New materials are used as sacrificial materials in vascular channel printing. Miller et al printed rigid 3D filament networks of carbohydrate glass, and subsequently the cell-laden hydrogel was casted to form tissues of several millimeters to centimeters (Figure 2D). The carbohydrate glass served as a cytocompatible sacrificial template and was dissolved after the culture medium was added. The engineered vascular networks were lined with endothelial cells and perfused with blood under high-pressure pulsatile flow. The perfused vascular channels sustained the metabolic function of primary rat hepatocytes in engineered tissue constructs. ${ }^{61}$ Other approaches of vascularization include micro-patterning and cell self-assembling process (similar to vasculogenesis). For example, the fabrication of microchannels in an alginate scaffold using a laser engraving system also promoted vessel-like networks and increased cell penetration and vessel density after subcutaneous implantation in mice. ${ }^{62}$ In another study, inkjet-printed scaffolds with human AFS cells, canine smooth muscle cells, and bovine aortic endothelial cells survived and 
matured into tissues with vascularization when implanted subcutaneously into athymic nude mice for up to 18 weeks. ${ }^{63}$ In addition, vascularized adipose tissue was generated in vivo by adipocyte hydrogel microspheres fabricated by a noncontact microfabrication device. After 4 weeks in an in vitro culture and then injected subcutaneously into nude mice, the regenerated vasculature showed functional anastomosis with host blood vessels. ${ }^{64}$

However, integrating vascular hierarchical structures spanning arteries down to capillaries has remained elusive. Lee et al developed a bioprinting technology $y^{53,65-68}$ to create perfused functional vascular channels $(0.5-1 \mathrm{~mm})$ with open lumen and vascular endothelial lining within collagen matrix using only cells and biological matrices. ${ }^{65}$ The fabricated vasculature has a tight, confluent endothelium lining, presenting barrier function. The vascular channel is capable of supporting the viability of a tissue up to $5 \mathrm{~mm}$ in distance at $5 \times 10^{6}$ cells $/ \mathrm{mL}$ density under the physiological flow condition. To create a multi-scale vascular network, they developed a 3D printing method to construct larger (lumen size of $\sim 1$ $\mathrm{mm}$ ) vascular channels and to create an adjacent capillary network through a natural maturation process, thus providing a feasible solution to connect the capillary network to the large perfused vascular channels. In the model, microvascular bed was formed in between two large fluidic vessels and then connected to the vessels by angiogenic sprouting from the large channel edge. ${ }^{69}$

\section{Nerve}

To facilitate nerve regeneration, hydrogel can be incorporated with the embedded growth factors during bioprinting, which would enable a slow release of the factors to promote neural regeneration. In one study, neural stem cells, collagen hydrogel, and vascular endothelial growth factor (VEGF)releasing fibrin gel were printed to construct an artificial neural tissue. The neural stem cells printed within $1 \mathrm{~mm}$ from the border of the VEGF-releasing fibrin gel showed VEGFinduced changes in their morphology and migrated toward the fibrin gel. The results demonstrated that bioprinting of VEGF-containing fibrin gel supported sustained release of the growth factor in the collagen scaffold. This method can be used in the development of 3D artificial tissue assays and neural tissue regeneration applications. ${ }^{6}$

In designing tissue-engineered nerve graft, multi-luminal channels can be incorporated in the design to potentially facilitate axon regrowth. The 3D bioprinting technology can be used to create nerve graft with multi-lumen channels. Owens et al developed a bioprinting approach to biofabricate fully biological grafts composed exclusively of cells and cell-secreted material. In this study, the MSCs and Schwann cells were mixed and used to create the cellular cylinders $(\sim 500 \mu \mathrm{m})$. These rods were then layered to create structures with multi-lumen channels using the bioprinting technology. The biological graft formed post-printing, by the fusion of the bioink cylinders. After a maturation period of 7 days, the multichannel construct developed sufficient mechanical integrity to be implanted into laboratory rats. This result demonstrated that bioprinting is a promising approach to nerve graft fabrication and as a consequence to nerve regeneration. ${ }^{70}$

\section{Trachea}

The flexibility of 3D bioprinting makes it particularly useful to create tracheas that match the anatomical geometry and shape. A 3D-printed scaffold was used for tracheal reconstruction in an in vivo tracheal defect model. First, 3D-printed PCL scaffold was coated with MSCs seeded in fibrin. The tracheal graft was implanted on a $10 \times 10 \mathrm{~mm}^{2}$ artificial tracheal defect in rabbits. After 4 and 8 weeks, it was demonstrated that trachea was successfully reconstructed without any collapse or blockage. The bioprinted trachea undergoes remodeling and was covered with regenerated respiratory mucosa. Histologic analysis showed that the implanted 3D-printed tracheal grafts were successfully integrated with the adjacent tracheal tissues without disruption or granulation tissue formation. The mechanical property of the newly generated neocartilage inside the implanted graft was strong enough to maintain the structural integrity of the reconstructed trachea. Functionally, the 3D-printed PCL trachea integrated with the adjacent tracheal tissues and allowed basic respiratory functions..$^{38,71}$

The combination of $3 \mathrm{D}$ bioprinting and medical imaging can be used to create patient-specific tissues for implantation. In a recent study, a customized, bioresorbable tracheal splint made of PCL was created by laser-based 3D printing with a computer-aided design based on a computed tomography (CT) image of the patient's airway. One year after the surgery, imaging and endoscopy showed normal structure and function in a patent left mainstem bronchus. This study illustrated the potential of $3 \mathrm{D}$ bioprinting in combination with $3 \mathrm{D}$ imaging to create patient-specific, anatomically correct tissue engineering constructs. ${ }^{72}$

\section{Cardiac tissue}

Application of 3D bioprinting in cardiac tissue regeneration is still in very early stages. There is very little success in this area due to many significant technical and biological 
challenges. In one study, human umbilical vascular endothelial cells and MSCs were seeded in a defined pattern on a polyester-urethane-urea cardiac patch fabricated by the laser-induced forward transfer cell printing technique. The patches were transplanted to the infarcted zone of rat hearts after ligation, leading to increased vessel formation, capillary density, and integration of human cells into the functionally connected vessels of the host vascular system. ${ }^{73}$ To repair myocardial tissue, Gaetani et al fabricated structures composed of a mixture of human cardiomyocyte progenitor cells and alginate hydrogel by bioprinting. In an in vivo study, the printed cells retained their commitment for the cardiac lineage and expressed the genes of the early cardiac transcription factors. ${ }^{74}$

\section{Heart valve}

Heart valve is an anatomically complex structure, which is particularly suitable for 3D bioprinting technology. Microextrusion bioprinters have been instrumental in the fabrication of aortic valves (Figure 2E). ${ }^{75,76}$ Duan et al applied an extrusion-based bioprinting technology into the construction of a trileaflet heart valve conduit, composed of hybrid hydrogel of hyaluronic acid and gelatin and human aortic valve interstitial cells. ${ }^{77}$ This study showed that the printed trileaflet heart valve conduit, assessed at 7 days, is highly viable and has great potential for remodeling. Subsequent study fabricated an anatomically complex living aortic valve conduit using alginate/gelatin hydrogel containing aortic root sinus smooth muscle cells and aortic valve interstitial cells. These studies suggest that it is feasible to create cellularized tissue valves using the bioprinting technology for eventual clinical use. ${ }^{75,77}$

\section{Future perspective}

Bioprinting has shown great promise in engineering 3D tissues with various cells and biomaterials. Despite having immense potential and being relatively simple in terms of architectures, components, and biological functions, only a few of the bioprinted tissues have achieved the fully functional states. In the clinical translation area, studies are primarily on bone, cartilage, tracheal, teeth, and skin regeneration. Few preclinical studies have been conducted for the regeneration of internal organs such as the lungs, kidneys, liver, and heart. Many challenges need to be overcome for broad in vivo applications of $3 \mathrm{D}$ bioprinting. Here, we review some of the most critical challenges in the $3 \mathrm{D}$ bioprinting field, including engineering challenges, biomaterial challenges, and biological challenges. We also propose some approaches to overcome these challenges.

\section{Engineering issues}

All the current bioprinting technologies, such as inkjet, microextrusion, and laser bioprinting, have their own advantages and disadvantages. Each of them is suitable for a particular type of materials. Currently, there is no unified technology that is compatible with many different materials for different tissues. There is also a need to develop bioprinting technologies to improve the resolution, speed, reproducibility, viability, and biocompatibility of the bioprinting processes. It is much needed to design new dispensing technologies to handle specific biological components instead of modification of preexisting technologies; thus, the range of compatible materials can be extended with increasing speed, precision, and specificity. For clinical usage, it is also necessary to increase the speed of fabrication for generating large-size construct. One method to achieve this would be to combine different printing technologies. For example, microextrusion can be combined with inkjet or laser bioprinting to generate macroscale tissue blocks that contain microscale features without taking too long time. To simultaneously handle multiple different types of materials and cells, we need to integrate a fully automatic, multi-modal, multi-material 3D bioprinting technology that is compatible with a diverse range of biomaterials and cells, and offer multi-scale control ( $\mu \mathrm{m}-\mathrm{mm})$ of cells and matrix. This can be achieved by combining the unique advantages of each of the current printing techniques, while avoiding the drawbacks of them. Besides printing technologies, lack of precise and real-time control of the printing environment (humidity, temperature, $\mathrm{CO}_{2}, \mathrm{O}_{2}, \mathrm{UV}$, etc) is still common in current systems. Most cross-linking and gelation procedures are still done manually. Therefore, it is also important to develop such real-time automatic controls during the printing process. To do this, it is also important to research and establish the printability database for various materials, standardize printing parameters, and build them into the printing control software. With the integration of an array of technologies into the printing process, the system will undoubtedly become extremely technically complex and more expensive, which will prevent it from being accessed by many tissue engineers and biomedical researchers. Hence, developing a simplified system and lowering the cost for commercialization are important tasks in this field. 


\section{Biomaterial issues}

Biomaterials play an essential role in any current and future bioprinting technologies. The materials connect the bioprinting process and the biological components, and act as the supporting system and the regulator for the post-printing tissue maturation. Current choices of printable biomaterials (based on rheological properties and cross-linking mechanisms) are very limited. Few biomaterials exist that both integrate seamlessly with bioprinting hardware and possess all the desired mechanical and biological properties. It is also very difficult to achieve the native ECM compositions/architecture, which have complex combinations and gradients of many ECM components, each with specific biological and mechanical properties. Therefore, it is much needed to develop new biomaterials that can be easily manipulated by the bioprinting technology to be dispensed in complex 3D structures and to maintain cellular viability and function, including new cytocompatible cross-link/gelation mechanisms. From the basic science point of view, we also need to improve the understanding of the matrix environment of various tissues, which are drastically different from each other. These also include developing methods to image, map the physiological ECMs, and establish methods to reproduce the ECM compositions of native tissues (eg, brain, liver, pancreas, lung, etc), to mimic the native ECM environments. It may be necessary to adapt different printing technologies and biomaterials for different tissues. While developing materials that can replicate the entire ECM compositions of a particular tissue is a daunting task, decellularized ECM-derived bioink is a more feasible approach for bioprinting applications. It may be needed to establish a decellularized ECM bioink library for each tissue. This set of materials, together with bioprinting technologies, can be used to create living structures customized for specific tissue regeneration.

\section{Biological issues}

There are many biological issues related to 3D bioprinting. One of them is the cell source and quantity. It is required to have a well-characterized and reproducible source of cells with the desired functions and be readily available in large quantities. For most tissues, it is also required to print multiple cell types simultaneously in order to achieve the desired function; hence, we need to have a greater understanding of the heterogeneous cell types and their interactions in the tissues. Compared with the fully differentiated adult cells such as chondrocytes, usage of stem cells needs additional knowledge on how to control their differentiation toward the desired lineages. To control stem cell differentiation in the bioprinting process, small molecules or growth factors can be incorporated in the bioprinting process. For the long-term viability of any bioprinted tissue construct, cell survival and vascularization within the printed structures are central issues. Oxygen, nutrients, and metabolic waste products are transported via a microvascular and an interstitial network that are connected to the systemic circulation. Angiogenesis can be promoted by pre-seeding printed scaffolds with mature or precursor endothelial cells or by the incorporation of angiogenic growth factors. Additionally, scaffolds can be designed to have pore size, porosity, and pore size distribution that favor vascular sprouting. To achieve the desired mechanical properties and functions of a bioprinted tissue, it is also required to undergo tissue maturation in bioreactors. Challenges will be to develop bioreactors that best mimic the biomechanical and biochemical environments of the in vivo situation. The bioreactor parameters such as temperature, $\mathrm{pH}$, nutrient, and gas concentrations as well as regulation of specific mechanical stimulations will depend on the bioreactor design, which is specific for each tissue type, and developmental goal. 3D-bioprinted tissue constructs are being developed not only for tissue regeneration but also for use in drug discovery, toxicity screening, and basic research. For these applications, extensive validations are needed to ensure that the bioprinted tissues can recapitulate the key pathophysiological features of the disease models. Finally, there is still a lack of translating 3D printing techniques from bench to bedside. This is attributed to a number of factors. Few biomaterial scientists and engineers have partnered with surgeons or scientists who are capable of performing preclinical and/or clinical studies. Technical factors that have precluded the application of $3 \mathrm{D}$ printing techniques in preclinical and/or clinical studies include cell survival and directed differentiation requirements, the fidelity of operating steps, as well as insufficient rate of vascularization and diffusion of metabolites. The close collaboration and formation of multidisciplinary teams among bioengineers, biologists, and doctors will be essential to meet these challenges and realize the full potential of $3 \mathrm{D}$ bioprinting technology.

\section{Acknowledgments}

We acknowledge the support from NSF CBET-1263455, NSF Career-1350240, NIH R01HL118245, and American Heart Association 12SDG12050083. 


\section{Disclosure}

The authors report no conflicts of interest in this work.

\section{References}

1. Hollister SJ. Porous scaffold design for tissue engineering. Nat Mater. 2005;4(7):518-524.

2. Giordano RA, Wu BM, Borland SW, Cima LG, Sachs EM, Cima MJ. Mechanical properties of dense polylactic acid structures fabricated by three dimensional printing. J Biomater Sci Polym Ed. 1996;8(1): 63-75.

3. Yang S, Leong KF, Du Z, Chua CK. The design of scaffolds for use in tissue engineering. Part II. Rapid prototyping techniques. Tissue Eng. 2002;8(1):1-11.

4. Lin CY, Kikuchi N, Hollister SJ. A novel method for biomaterial scaffold internal architecture design to match bone elastic properties with desired porosity. J Biomech. 2004;37(5):623-636.

5. Hutmacher DW, Sittinger M, Risbud MV. Scaffold-based tissue engineering: rationale for computer-aided design and solid free-form fabrication systems. Trends Biotechnol. 2004;22(7):354-362.

6. Peltola SM, Melchels FP, Grijpma DW, Kellomaki M. A review of rapid prototyping techniques for tissue engineering purposes. Ann Med. 2008;40(4):268-280.

7. Sun W, Darling A, Starly B, Nam J. Computer-aided tissue engineering: overview, scope and challenges. Biotechnol Appl Biochem. 2004;39(Pt 1): 29-47.

8. Yeong WY, Chua CK, Leong KF, Chandrasekaran M. Rapid prototyping in tissue engineering: challenges and potential. Trends Biotechnol. 2004;22(12):643-652.

9. Norotte C, Marga FS, Niklason LE, Forgacs G. Scaffold-free vascular tissue engineering using bioprinting. Biomaterials. 2009;30(30):5910-5917.

10. Mironov V, Visconti RP, Kasyanov V, Forgacs G, Drake CJ, Markwald RR. Organ printing: tissue spheroids as building blocks. Biomaterials. 2009;30(12):2164-2174.

11. Mironov V, Boland T, Trusk T, Forgacs G, Markwald RR. Organ printing: computer-aided jet-based 3D tissue engineering. Trends Biotechnol. 2003;21(4):157-161.

12. Roth EA, Xu T, Das M, Gregory C, Hickman JJ, Boland T. Inkjet printing for high-throughput cell patterning. Biomaterials. 2004;25(17):3707-3715.

13. Xu T, Jin J, Gregory C, Hickman JJ, Boland T. Inkjet printing of viable mammalian cells. Biomaterials. 2005;26(1):93-99.

14. Dhariwala B, Hunt E, Boland T. Rapid prototyping of tissue-engineering constructs, using photopolymerizable hydrogels and stereolithography. Tissue Eng. 2004;10(9-10):1316-1322.

15. Landers R, Hubner U, Schmelzeisen R, Mulhaupt R. Rapid prototyping of scaffolds derived from thermoreversible hydrogels and tailored for applications in tissue engineering. Biomaterials. 2002;23(23): 4437-4447.

16. Liu Tsang V, Chen AA, Cho LM, et al. Fabrication of 3D hepatic tissues by additive photopatterning of cellular hydrogels. FASEB J. 2007;21(3): 790-801.

17. Murphy SV, Atala A. 3D bioprinting of tissues and organs. Nat Biotechnol. 2014;32(8):773-785.

18. Cui X, Dean D, Ruggeri ZM, Boland T. Cell damage evaluation of thermal inkjet printed Chinese hamster ovary cells. Biotechnol Bioeng. 2010;106(6):963-969.

19. Xu T, Gregory CA, Molnar P, et al. Viability and electrophysiology of neural cell structures generated by the inkjet printing method. Biomaterials. 2006;27(19):3580-3588.

20. Tasoglu S, Demirci U. Bioprinting for stem cell research. Trends Biotechnol. 2013;31(1):10-19.

21. Demirci U, Montesano G. Single cell epitaxy by acoustic picolitre droplets. Lab Chip. 2007;7(9):1139-1145.

22. Lee W, Debasitis JC, Lee VK, et al. Multi-layered culture of human skin fibroblasts and keratinocytes through three-dimensional freeform fabrication. Biomaterials. 2009;30(8):1587-1595.
23. Singh $M$, Haverinen HM, Dhagat $P$, Jabbour GE. Inkjet printing-process and its applications. Adv Mater. 2010;22(6):673-685.

24. Cui X, Boland T. Human microvasculature fabrication using thermal inkjet printing technology. Biomaterials. 2009;30(31):6221-6227.

25. Khalil S, Sun W. Bioprinting endothelial cells with alginate for 3D tissue constructs. J Biomech Eng. 2009;131(11):111002.

26. Jakab K, Neagu A, Mironov V, Markwald RR, Forgacs G. Engineering biological structures of prescribed shape using self-assembling multicellular systems. Proc Natl Acad Sci U S A. 2004;101(9):2864-2869.

27. Chang R, Nam J, Sun W. Effects of dispensing pressure and nozzle diameter on cell survival from solid freeform fabrication-based direct cell writing. Tissue Eng Part A. 2008;14(1):41-48.

28. Schiele NR, Corr DT, Huang Y, Raof NA, Xie Y, Chrisey DB. Laser-based direct-write techniques for cell printing. Biofabrication. 2010;2(3):032001.

29. Guillotin B, Souquet A, Catros S, et al. Laser assisted bioprinting of engineered tissue with high cell density and microscale organization. Biomaterials. 2010;31(28):7250-7256.

30. Guillemot F, Souquet A, Catros S, et al. High-throughput laser printing of cells and biomaterials for tissue engineering. Acta Biomater. 2010;6(7):2494-2500.

31. Skardal A, Atala A. Biomaterials for integration with 3-D bioprinting. Ann Biomed Eng. 2015;43(3):730-746.

32. Murphy SV, Skardal A, Atala A. Evaluation of hydrogels for bio-printing applications. J Biomed Mater Res A. 2013;101(1):272-284

33. Bertassoni LE, Cardoso JC, Manoharan V, et al. Direct-write bioprinting of cell-laden methacrylated gelatin hydrogels. Biofabrication. 2014; 6(2):024105.

34. Seol YJ, Kang HW, Lee SJ, Atala A, Yoo JJ. Bioprinting technology and its applications. Eur J Cardiothorac Surg. 2014;46(3):342-348.

35. Guillemot F, Mironov V, Nakamura M. Bioprinting is coming of age: report from the International Conference on Bioprinting and Biofabrication in Bordeaux (3B'09). Biofabrication. 2010;2(1):010201.

36. Pashuck ET, Stevens MM. Designing regenerative biomaterial therapies for the clinic. Sci Transl Med. 2012;4(160):160sr164.

37. Bajaj P, Schweller RM, Khademhosseini A, West JL, Bashir R. 3D biofabrication strategies for tissue engineering and regenerative medicine. Annu Rev Biomed Eng. 2014;16:247-276.

38. Park JH, Jung JW, Kang HW, Joo YH, Lee JS, Cho DW. Development of a $3 \mathrm{D}$ bellows tracheal graft: mechanical behavior analysis, fabrication and an in vivo feasibility study. Biofabrication. 2012;4(3):035004.

39. Pati F, Jang J, Ha DH, et al. Printing three-dimensional tissue analogues with decellularized extracellular matrix bioink. Nat Commun. 2014;5: 3935.

40. Li J, He L, Zhou C, et al. 3D printing for regenerative medicine: from bench to bedside. MRS Bull. 2015;40(2):145-153.

41. Strobel LA, Rath SN, Maier AK, et al. Induction of bone formation in biphasic calcium phosphate scaffolds by bone morphogenetic protein-2 and primary osteoblasts. J Tissue Eng Regen Med. 2014; 8(3):176-185.

42. Weinand C, Gupta R, Weinberg E, et al. Toward regenerating a human thumb in situ. Tissue Eng Part A. 2009;15(9):2605-2615.

43. Temple JP, Hutton DL, Hung BP, et al. Engineering anatomically shaped vascularized bone grafts with hASCs and 3D-printed PCL scaffolds. J Biomed Mater Res A. 2014;102(12):4317-4325.

44. Fedorovich NE, Wijnberg HM, Dhert WJ, Alblas J. Distinct tissue formation by heterogeneous printing of osteo- and endothelial progenitor cells. Tissue Eng Part A. 2011;17(15-16):2113-2121.

45. Xu T, Binder KW, Albanna MZ, et al. Hybrid printing of mechanically and biologically improved constructs for cartilage tissue engineering applications. Biofabrication. 2013;5(1):015001.

46. Cui X, Breitenkamp K, Finn MG, Lotz M, D’Lima DD. Direct human cartilage repair using three-dimensional bioprinting technology. Tissue Eng Part A. 2012;18(11-12):1304-1312.

47. Cui X, Breitenkamp K, Lotz M, D'Lima D. Synergistic action of fibroblast growth factor-2 and transforming growth factor-beta1 enhances bioprinted human neocartilage formation. Biotechnol Bioeng. 2012; 109(9):2357-2368. 
48. Fedorovich NE, Schuurman W, Wijnberg HM, et al. Biofabrication of osteochondral tissue equivalents by printing topologically defined, cell-laden hydrogel scaffolds. Tissue Eng Part C Methods. 2012;18(1): 33-44.

49. Lee CH, Marion NW, Hollister S, Mao JJ. Tissue formation and vascularization in anatomically shaped human joint condyle ectopically in vivo. Tissue Eng Part A. 2009;15(12):3923-3930.

50. Gurkan UA, El Assal R, Yildiz SE, et al. Engineering anisotropic biomimetic fibrocartilage microenvironment by bioprinting mesenchymal stem cells in nanoliter gel droplets. Mol Pharm. 2014;11(7):2151-2159.

51. Lee CH, Cook JL, Mendelson A, Moioli EK, Yao H, Mao JJ. Regeneration of the articular surface of the rabbit synovial joint by cell homing: a proof of concept study. Lancet. 2010;376(9739): 440-448.

52. Mendelson A, Frank E, Allred C, et al. Chondrogenesis by chemotactic homing of synovium, bone marrow, and adipose stem cells in vitro. FASEB J. 2011;25(10):3496-3504.

53. Lee V, Singh G, Trasatti JP, et al. Design and fabrication of human skin by three-dimensional bioprinting. Tissue Eng Part C Methods. 2014;20(6):473-484.

54. Koch L, Deiwick A, Schlie S, et al. Skin tissue generation by laser cell printing. Biotechnol Bioeng. 2012;109(7):1855-1863.

55. Michael S, Sorg H, Peck CT, et al. Tissue engineered skin substitutes created by laser-assisted bioprinting form skin-like structures in the dorsal skin fold chamber in mice. PLoS One. 2013;8(3):e57741.

56. Skardal A, Mack D, Kapetanovic E, et al. Bioprinted amniotic fluidderived stem cells accelerate healing of large skin wounds. Stem Cells Transl Med. 2012;1(11):792-802.

57. Lee W, Lee V, Polio S, et al. On-demand three-dimensional freeform fabrication of multi-layered hydrogel scaffold with fluidic channels. Biotechnol Bioeng. 2010;105(6):1178-1186.

58. Skardal A, Zhang J, McCoard L, Xu X, Oottamasathien S, Prestwich GD. Photocrosslinkable hyaluronan-gelatin hydrogels for two-step bioprinting. Tissue Eng Part A. 2010;16(8):2675-2685.

59. Kolesky DB, Truby RL, Gladman AS, Busbee TA, Homan KA, Lewis JA. 3D bioprinting of vascularized, heterogeneous cell-laden tissue constructs. Adv Mater. 2014;26(19):3124-3130.

60. Bertassoni LE, Cecconi M, Manoharan V, et al. Hydrogel bioprinted microchannel networks for vascularization of tissue engineering constructs. Lab Chip. 2014;14(13):2202-2211.

61. Miller JS, Stevens KR, Yang MT, et al. Rapid casting of patterned vascular networks for perfusable engineered three-dimensional tissues. Nat Mater. 2012;11(9):768-774.

62. Zieber L, Or S, Ruvinov E, Cohen S. Microfabrication of channel arrays promotes vessel-like network formation in cardiac cell construct and vascularization in vivo. Biofabrication. 2014;6(2):024102.

63. Xu T, Zhao W, Zhu JM, Albanna MZ, Yoo JJ, Atala A. Complex heterogeneous tissue constructs containing multiple cell types prepared by inkjet printing technology. Biomaterials. 2013;34(1):130-139.
64. Yao R, Zhang R, Lin F, Luan J. Injectable cell/hydrogel microspheres induce the formation of fat lobule-like microtissues and vascularized adipose tissue regeneration. Biofabrication. 2012;4(4):045003.

65. Lee VK, Kim DY, Ngo H, et al. Creating perfused functional vascular channels using 3D bio-printing technology. Biomaterials. 2014;35(28): 8092-8102.

66. Zhao L, Lee VK, Yoo SS, Dai G, Intes X. The integration of 3-D cell printing and mesoscopic fluorescence molecular tomography of vascular constructs within thick hydrogel scaffolds. Biomaterials. 2012; 33(21):5325-5332.

67. Ozturk MS, Lee VK, Zhao L, Dai G, Intes X. Mesoscopic fluorescence molecular tomography of reporter genes in bioprinted thick tissue. J Biomed Opt. 2013;18(10):100501.

68. Lee YB, Polio S, Lee W, et al. Bio-printing of collagen and VEGFreleasing fibrin gel scaffolds for neural stem cell culture. Exp Neurol. 2010;223(2):645-652.

69. Lee VK, Lanzi AM, Haygan N, Yoo SS, Vincent PA, Dai G. Generation of multi-scale vascular network system within 3D hydrogel using 3d bio-printing technology. Cell Mol Bioeng. 2014;7(3):460-472.

70. Owens CM, Marga F, Forgacs G, Heesch CM. Biofabrication and testing of a fully cellular nerve graft. Biofabrication. 2013;5(4):045007.

71. Chang JW, Park SA, Park JK, et al. Tissue-engineered tracheal reconstruction using three-dimensionally printed artificial tracheal graft: preliminary report. Artif Organs. 2014;38(6):E95-E105.

72. Zopf DA, Hollister SJ, Nelson ME, Ohye RG, Green GE. Bioresorbable airway splint created with a three-dimensional printer. $N$ Engl J Med. 2013;368(21):2043-2045.

73. Gaebel R, Ma N, Liu J, et al. Patterning human stem cells and endothelial cells with laser printing for cardiac regeneration. Biomaterials. 2011; 32(35):9218-9230.

74. Gaetani R, Doevendans PA, Metz CH, et al. Cardiac tissue engineering using tissue printing technology and human cardiac progenitor cells. Biomaterials. 2012;33(6):1782-1790.

75. Duan B, Hockaday LA, Kang KH, Butcher JT. 3D bioprinting of heterogeneous aortic valve conduits with alginate/gelatin hydrogels. J Biomed Mater Res A. 2013;101(5):1255-1264.

76. Hockaday LA, Kang KH, Colangelo NW, et al. Rapid 3D printing of anatomically accurate and mechanically heterogeneous aortic valve hydrogel scaffolds. Biofabrication. 2012;4(3):035005.

77. Duan B, Kapetanovic E, Hockaday LA, Butcher JT. Three-dimensional printed trileaflet valve conduits using biological hydrogels and human valve interstitial cells. Acta Biomater. 2014;10(5):1836-1846.

78. Gao G, Yonezawa T, Hubbell K, Dai G, Cui X. Inkjet-bioprinted acrylated peptides and PEG hydrogel with human mesenchymal stem cells promote robust bone and cartilage formation with minimal printhead clogging. Biotechnol J. Epub January 8, 2015.

79. Cui X, Gao G, Yonezawa T, Dai G. Human cartilage tissue fabrication using three-dimensional inkjet printing technology. JVis Exp. 2014;88: $475-481$.
Advanced Health Care Technologies

\section{Publish your work in this journal}

Advanced Health Care Technologies is an international, peer reviewed, open access journal that provides a unique forum for articles on: point-of-care, health care diagnostics and treatment, bioengineering, biotechnology, biosensing, electronics, clinical/medical science, chemical engineering, materials science, regenerative medicine, micro-/

\section{Dovepress}

nano-technologies, and methods and applications for nanoscience and nanotechnology. The manuscript management system is completely online and includes a very quick and fair peer review system, which is all easy to use. Visit http://www.dovepress.com/testimonials.php to read real quotes from published authors. 